\title{
First-principles investigation of the impact of high pressure on the structural, electronic and elastic properties of the type-VIII barium-doped silicon clathrate $\mathrm{Ba}_{8} \mathrm{Si}_{46}$.
}

\author{
Nassim Ahmed MAHAMMEDI ${ }^{1,2}$, Marhoun FERHAT ${ }^{2,3}$ \\ ${ }^{1}$ Laboratoire de physique des matériaux LPM, Amar Télidji University of Laghouat, BP37G, Laghouat 03000, ALGERIA. \\ ${ }^{2}$ École Normale Supérieure de Laghouat (ENSL),RN01, Laghouat 03000, ALGERIA. \\ ${ }^{3}$ Department of physics, The University of the West Indies, Mona, Kingston 07, JAMAICA. \\ Corresponding author \\ Nassim Ahmed MAHAMMEDI \\ n.mahammedi@lagh-univ.dz
}

\section{Abstract}

By means of density functional theory (DFT) calculations, we have investigated the effect of hydrostatic pressure on the structural, electronic and elastic properties of the barium-doped silicon clathrate $\mathrm{Ba}_{8} \mathrm{Si}_{46}$ in the type-VIII structure ( $\alpha$ phase). Physical properties are calculated under different conditions of pressure ( $0 \mathrm{GPa}$ to $45 \mathrm{GPa}$ ) using the GGA-PBE functional, those calculations have been performed using the Cambridge serial total energy package CASTEP code within the Materials Studio package. Electronic properties have shown that the type-VIII $\mathrm{Ba}_{8} \mathrm{Si}_{46}$ has metal-like properties with a fundamental bandgap of $1 \mathrm{eV}$. Under pressure the fundamental bandgap increases slightly and the positions of the valence band maximum VBM and the conduction band minimum CBM remain unchanged. We found that the compound is mechanically stable under the pressure range, but this needs to be confirmed experimentally through synthesis, a comparison with the type-I and the guest-free counterparts has exhibited promising features for the type-VIII $\mathrm{Ba}_{8} \mathrm{Si}_{46}$.

\section{Introduction}

The quest for a perfect semiconductor that combines several features is a challenging task and a revived competitive scientific topic for materials scientists and engineers. Silicon is one of the most investigated, handled, and utilized semiconductor in major high-tech devices including not only optics, electronics, microelectronics and photovoltaics, but also thermoelectrics, superconducting and future quantum computing devices. Additionally to the fact that silicon is the second abundant substance on the Earth's crust (about 25.8\% of its global mass), it has several advantages that made its industry mature and very advanced. However, exploring crystalline phases beyond the cubic diamond structure will certainly overcome its several shortages and provide more technological alternatives for expensive and rare semiconductors. Silicon clathrates are regarded as the most promising structures of silicon, they were first synthesized in 1965 by Kasper et al and Cros et al [1,2], and their discovery released prominent 
research for exploring and exploiting their many intriguing features including thermoelectrics [3, 4], electronics, photovoltaics [5], superconductors [6, 7], and even as anodes for Li-based batteries [8]. Si clathrates are cage-like framework of silicon that entraps guest atoms inside. Relatively to their constituents and their chemical formula, a very large family of clathrates can be formed [9]. Etymologically, the term 'Clathrate' is derived from the Greek word 'Klethra' which originally means 'alder' [10] and more precisely from the Latin word 'Clathrus', meaning 'surrounded on all sides'[11], the term was used in the 1940s to describe clathrates of $\beta$ hydroquinone compounds by Powell, H.M. [12]. Theoretically, eight types of clathrates are described, and more clathrate-like forms can also be added [4], however, only three types are successfully synthesized so far, i.e. type-I, type-II and type-VIII. Type-I and type-VIII clathrates possess the same chemical formulas $\mathrm{X}_{8} \mathrm{Si}_{46}$. Guest free silicon clathrates are promising materials for semiconducting and optoelectronic applications, as well as for thermoelectrics. Synthesis of guest-free silicon clathrates is still a challenging task, where attempting to grow single crystals, powder, or thin films only succeeded for the type-II configuration $[13,14]$. Thin film Silz6 was recently grown over a (111) Si substrate [15]. Norouzzadeh et al extensively investigated the type-VIII gust-free $\mathrm{Si}_{46}$ by first-principles calculations, their results showed very promising thermoelectric features of this material [16-18].

In this work, first-principles calculations in the frame of the DFT within the GGA approach are employed to investigate the main structural features and the impact of hydrostatic pressure on the structural, electronic and elastic properties of type-VIII Ba8Si46 silicon clathrates, thus, this paper is organized as follows: the first section outlines the theoretical approach including our computational details and structural aspects of the clathrate, the second section discusses the electronic properties under pressure, and then the elastic properties under pressure are discussed in detail.

\section{Theoretical aspects}

\section{II.1. Computational procedures}

In this work, first-principles calculations within the density functional theory DFT [19, 20] are performed using the Cambridge Serial Total Energy Package (CASTEP) code [21]. Plane-wave based norm-conserving pseudopotentials [22] are used. The electronic configurations in these pseudopotentials are $\mathrm{Ba}:[\mathrm{Xe}] 6 \mathrm{~s}^{2}$ and $\mathrm{Si}:[\mathrm{Ne}] 3 \mathrm{~s}^{2} 3 \mathrm{p}^{2}$. The general gradient approximation with the Perdew-Burke-Erzenhorf GGA-PBE [23] approach is selected to estimate the exchange and correlation energy $\mathrm{XC}$. In order to ensure that the self-consistent convergence of the total energy reaches $10^{-6} \mathrm{eV} /$ atom, the kinetic plane-wave cut-off energy is set to $480 \mathrm{eV}$ and the first 
Peer-reviewed version available at Silicon 2019, 12; doi:10.1007/s12633-019-00145-1

irreducible Brillouin zone is sampled using a 6x6x6 Monkhorst-Pack scheme [24]. Structural optimization is primordial in order to get an equilibrium structure at a minimum total energy, for that purpose, the optimizations are being performed using the Broyden-Fletcher-GoldfarbShanno (BFGS) algorithm [25-28] and stopped until the Hellmann-Feynman interatomic forces reach $10^{-5} \mathrm{eV} / \AA$ and the pressure reach a minimum value of $0.001 \mathrm{GPa}$ for all calculations. All physical properties are calculated for equilibrium crystals optimized at minimum of the total energy.

\section{II.2. Crystalline structure}

Type-VIII silicon clathrates typically crystallize in the body centered cubic space group I4-3m $\mathrm{N}^{\circ} 217$ [29, 30], their frameworks are initially composed from associations of polyhedral cages of silicon as a host material encapsulating alkali-metals or earth-like guest species. The strongly distorted dodecahedron cages $\mathrm{Si}_{23}$ are the only type of polyhedron composing the type-VIII structure, the silicon atoms occupy the 2a, 8c, 12d and 24g Wyckoff positions, whereas, guests (barium for our case) occupy the $8 \mathrm{c}$ positions inside those cages. The host silicon atoms are bonded to each other through the strong $\mathrm{sp}^{3}$ hybridization, while the barium atoms have strong van der Walls interactions with their surrounding Si clusters (due to their relatively larger size). The unit lattice of $\mathrm{Ba}_{8} \mathrm{Si}_{46}$-VIII (illustrated in figure1), contains 54 atoms, is used as a basic model throughout this work. We have firstly performed a full structural optimization at zero pressure and minimum of total energy and interatomic forces, our results showed that the lattice constants are $a=b=c=10.395 \AA$ (expected to be slightly overestimated by GGA). The results are compared with the literature and summarized in the table 1, unfortunately, no experimental results for type-VIII Ba8Si46 are reported so far. Our calculated structural parameters are in a fair agreement with the available data; however, the slight differences are due to the different DFT codes solicited for each study. In what follows, the Si atoms at 2a, 8c, 12d, and $24 \mathrm{~g}$ are symbolized as $\mathrm{Si1}, \mathrm{Si} 2, \mathrm{Si} 3$ and $\mathrm{Si} 4$ respectively.
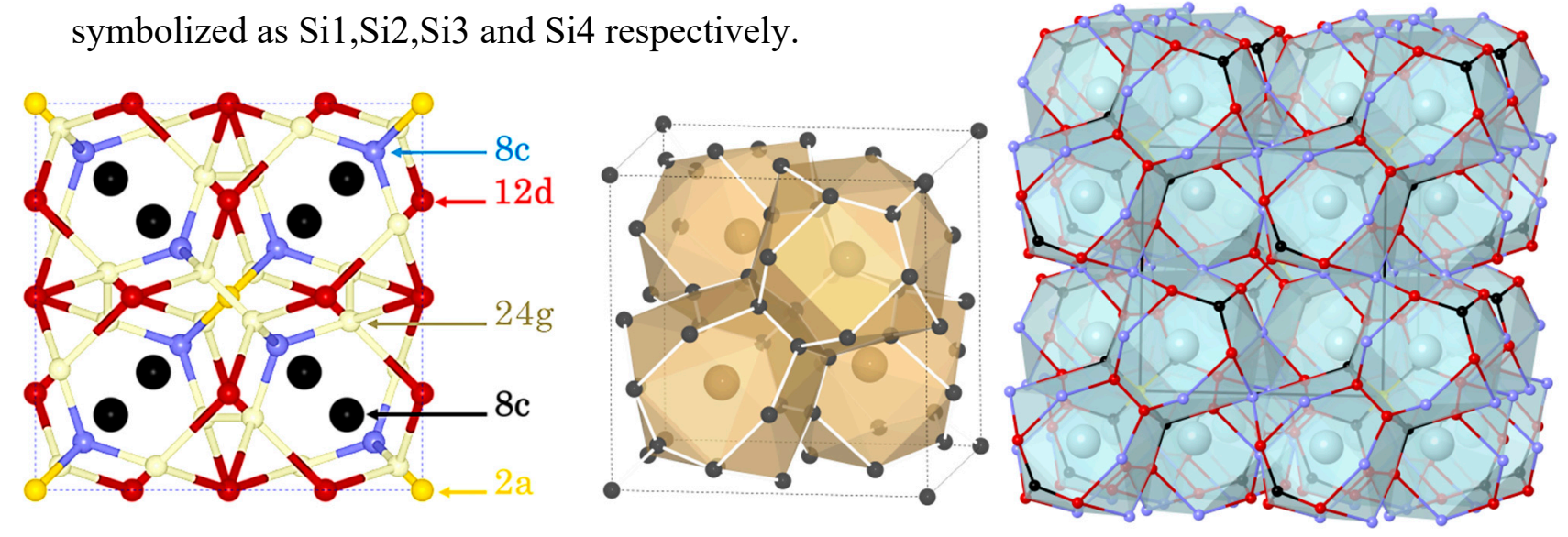
Peer-reviewed version available at Silicon 2019, 12; doi:10.1007/s12633-019-00145-1.

Figure 1. Aspects of the crystalline structures of the type-VIII Ba8Si46, (a) Wyckoff positions (b) and (c) elemental cages forming the unit lattice

\section{Results and discussions}

\section{III.1. Structural properties under high pressure}

In order to investigate the effect of hydrostatic pressure on the physical properties of VIII$\mathrm{Ba}_{8} \mathrm{Si}_{46}$, the equilibrium lattice constants at zero pressure and at minimum total energy are calculated and used as starting structural parameters for what follows. Our results for structural parameters including optimized lattice constants and atomic positions by GGA-PBE are summarized in the table 1 and are compared with available literature. It can be noticed that intercalation of barium atoms will increase the volume of the silicon clathrate network by $2 \%$; this is due to the relatively large size of Ba atoms. Experimentally, only ternary compounds of type-VIII clathrates have been reported, hence, experimental values for the type-VIII $\mathrm{Sr}_{8} \mathrm{Al}_{\mathrm{x}} \mathrm{Ga}_{\mathrm{y}} \mathrm{Si}_{46-\mathrm{x}-\mathrm{y}}$ (synthesized by means of arc-melting and spark plasma sintering SPS, $a=10.4444 \AA$ for $\mathrm{Sr}_{8} \mathrm{Al}_{10} \mathrm{Ga}_{6} \mathrm{Sr}_{30}$ ) by Shimizu et al [31], and for the type-I $\mathrm{Ba}_{8} \mathrm{Si}_{46}$ (synthesized by HPHT, $a=10.328 \AA$ ) by Yamanaka et al [32], are used for sake of comparison as well. Following structural optimization, we obtained lattice parameters at $0 \mathrm{GPa}$ as $a=10.395 \AA$ for type-VIII $\mathrm{Ba}_{8} \mathrm{Si}_{46}$ and $a=10.121 \AA$ for the guest free VIII-Si 46 . The lattice parameter of type-VIII Ba8Si46 obtained by GGA-PBE is closer to the experimental value of VIII-Sr8 $\mathrm{Al}_{10} \mathrm{Ga}_{6} \mathrm{Si}_{30}$ [31] and to that of the type-I Ba8Si46.Lattice parameters for the guest-free VIII-Si46 are lower than the mentioned experimental values, however, if compared to the experimental value of the guest-free type-II $\mathrm{Si}_{136}$ synthesized by Jan Gryko et al [13] (by means of successive vacuum treatment and density separation of $\mathrm{Na}_{\mathrm{x}} \mathrm{Si}_{136}$ ) (space group $\mathrm{Fd}-3 \mathrm{~m}, \mathrm{a}=14.62601 \AA$ ), the values are far different.

We calculated the Gibb's free energy at $0 \mathrm{GPa}$ using the formula $\mathbf{G}_{\mathbf{0}}=\mathbf{E}_{\mathbf{0}}+\mathbf{P V}-\mathbf{T}_{\mathbf{s}}$, where $\mathrm{E}_{0}$ is the total energy per unit cell and Ts is the temperature. Since this DFT study is held at a ground state where $T_{s}=0 \mathrm{~K}$, the Gibb's free energy will be equal to the enthalpy $\mathbf{H}_{\mathbf{0}}=\mathbf{E}_{\mathbf{0}}+\mathbf{p} \mathbf{V}_{\mathbf{0}}$. The Enthalpy variation with pressure is illustrated in figure2. Not surprisingly, it is observed that the most stable structure is under zero pressure, and then the enthalpy increases linearly with pressure above $5 \mathrm{GPa}$. Compared with the type-I phase, it was reported that the type-VIII $\mathrm{Ba}_{8} \mathrm{Ga}_{16} \mathrm{Sn}_{30}$ clathrate has lower enthalpy and therefore it tend to be more stable under high-pressure [33]. We also estimated the formation energy of the VIII-Bar $\mathrm{Si}_{46}$ from the following equation [16]:

$$
\Delta \mathbf{H}\left(\mathrm{M}_{8} \mathrm{Si}_{46}\right)=\mathrm{E}_{B a s S i 46}-8 \mathrm{E}_{M}-46 \mathrm{E}_{S i}
$$

Where: $\Delta \mathrm{H}$ is the total formation energy, $\mathrm{E}_{\mathrm{M}}$ is the total energy of the intercalated atom calculated in its stable bulk phase, and $\mathrm{E}_{\mathrm{Si}}$ is the total energy of Si atom in the stable diamond 
Peer-reviewed version available at Silicon 2019, 12; doi:10.1007/s12633-019-00145-1

phase. A negative value is supposed to indicate that the compound is energetically favorable to form, whereas, if it is positive, that means that the material could be synthesized but require an additional amount of energy. We report a value of $\Delta \mathrm{H}=-97.23 \mathrm{eV}$.

The evolution of the lattice volumes and the interatomic distances $\mathrm{Si}-\mathrm{Si}$ and $\mathrm{Ba}-\mathrm{Si}$ under highpressure are illustrated in figures 3 and 4 respectively. As can be noticed from figure 3 , the cell volume tend to normally decrease when pressure is increased, however, the behavior is not linear, from $0 \mathrm{GPa}$ to $5 \mathrm{GPa}$, the volume is drastically reduced by about $31 \%$. Above $10 \mathrm{GPa}$, lattice volume will decrease in a quasi-linear manner with compressive pressure. At 30 GPa the overall volume is reduced by about 49\%. According to Kim et al [34], a volume collapse above $20 \mathrm{GPa}$ is expected for the type-VIII quaternary substituted silicon clathrate $\mathrm{Sr}_{8} \mathrm{Al}_{8} \mathrm{Ga}_{8} \mathrm{Si}_{30}$, therefore, in our case, no such change is observed. Leoni et al [35] has suggested an analytical model to analyze the phase transition from type-I ( $\beta$ phase) to type-VIII ( $\alpha$ phase) clathrates. High pressure behavior for type-I Ba $\mathrm{Si}_{46}$ by Zhang et al [36] and $\mathrm{K}_{8} \mathrm{Si}_{46}$ by Tse et al [37] were studied by means of DFT codes. In both studies similar behaviors were observed with higher mechanical stability for the barium doped clathrate as a result of the larger $\mathrm{Ba}$ atoms and hence stronger interaction with the Si cage. A study by Li et al [33] on the high-pressure behavior of the type-VIII $\mathrm{Ba}_{8} \mathrm{Ga}_{16} \mathrm{Sn}_{30}$ has also predicted that the type-VIII clathrate is more stable than its type-I allotrope, and they found that the phase transition from type-I to type-VIII $(\beta \rightarrow \alpha)$ cannot occur under hydrostatic pressure regardless its magnitude. This will be understood in the discussion of mechanical properties section. Figure 3(a) exhibits the variation of the Ba-Si and Si-Si bonds under high pressure. Ba-Si and Si-Si bond lengths have different behaviors towards pressure, thus, the Ba-Si has similar tendencies towards lower values when pressure is increased. This monotonous behavior demonstrates the isotropic compression of the cages under pressure; this is certainly related to the enhanced integrity of the Si cages by the presence of $\mathrm{Ba}$ atoms. Nevertheless, generally, the expansion of cages volume caused by the presence of large Ba atoms will loosen the Si-Si bonding, even if the Ba-Si interaction contributes to the integrity of the structure but the Si cage will be weakened.

Si-Si bonds in contrary show different behaviors towards pressure, for example, the Si3(12d)$\mathrm{Si} 4(24 \mathrm{~g})$ and $\mathrm{Si} 4(24 \mathrm{~g})-\mathrm{Si} 4(24 \mathrm{~g})$ distances decrease slowly comparing to the $\mathrm{Si} 1(2 \mathrm{a})-\mathrm{Si} 2(8 \mathrm{c})$ and $\mathrm{Si} 2(8 \mathrm{c})-\mathrm{Si} 4(24 \mathrm{~g})$ bonds. It can be observed that the three Si-Si lengths converge towards similar values at $30 \mathrm{GPa}$ unlike the $\mathrm{Si1}(2 \mathrm{a})-\mathrm{Si} 2(8 \mathrm{c})$ bond which seems to decrease more rapidly.

We have also examined the evolution of angles in the silicon frameworks under high-pressure and their evolution is plotted in the figure 3(b). It can be seen that the $\mathrm{Si}(2-1-2)$ angle in the 
Peer-reviewed version available at Silicon 2019, 12; doi:10.1007/s12633-019-00145-1

pentagonal rings are constant under pressure. Other angles such as the Si(4-2-4) in the six-fold rings and the $\mathrm{Si}(4-3-4)$ in the seven-fold rings are not affected by pressure. The $\mathrm{Si}(4-3-4)$ in the six-fold rings increases with pressure, whereas, the $\mathrm{Si}(2-4-3)$ and $\mathrm{Si}(4-4-3)$ in the six- and sevenfold rings respectively decrease rapidly with pressure. At $20 \mathrm{GPa}$, it can be noticed that the $\operatorname{Si}(1$ 2-4) and the $\mathrm{Si}(2-4-4)$ in the pentagonal rings became equal and cross at this pressure. Anisotropic behavior of angles is a normal characteristic of cage-like structures and is a consequence of the isotropic behavior of the overall lattice volume.
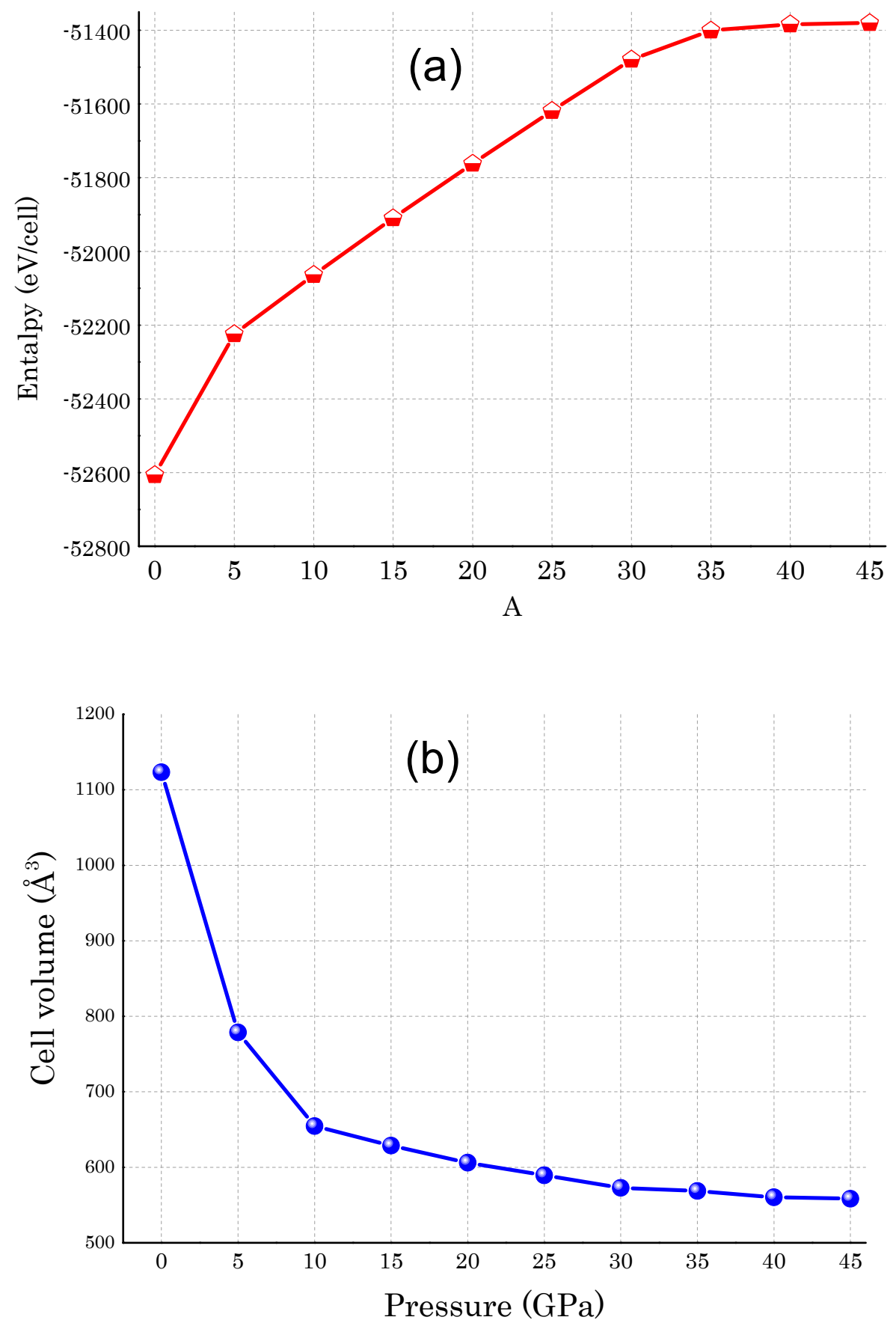

Figure 2. Evolution of (a) the Total enthalpy and (b) the equilibrium volume of VIII-Ba8 $\mathrm{Si}_{46}$ under pressure 
Peer-reviewed version available at Silicon 2019, 12; doi:10.1007/s12633-019-00145-1.
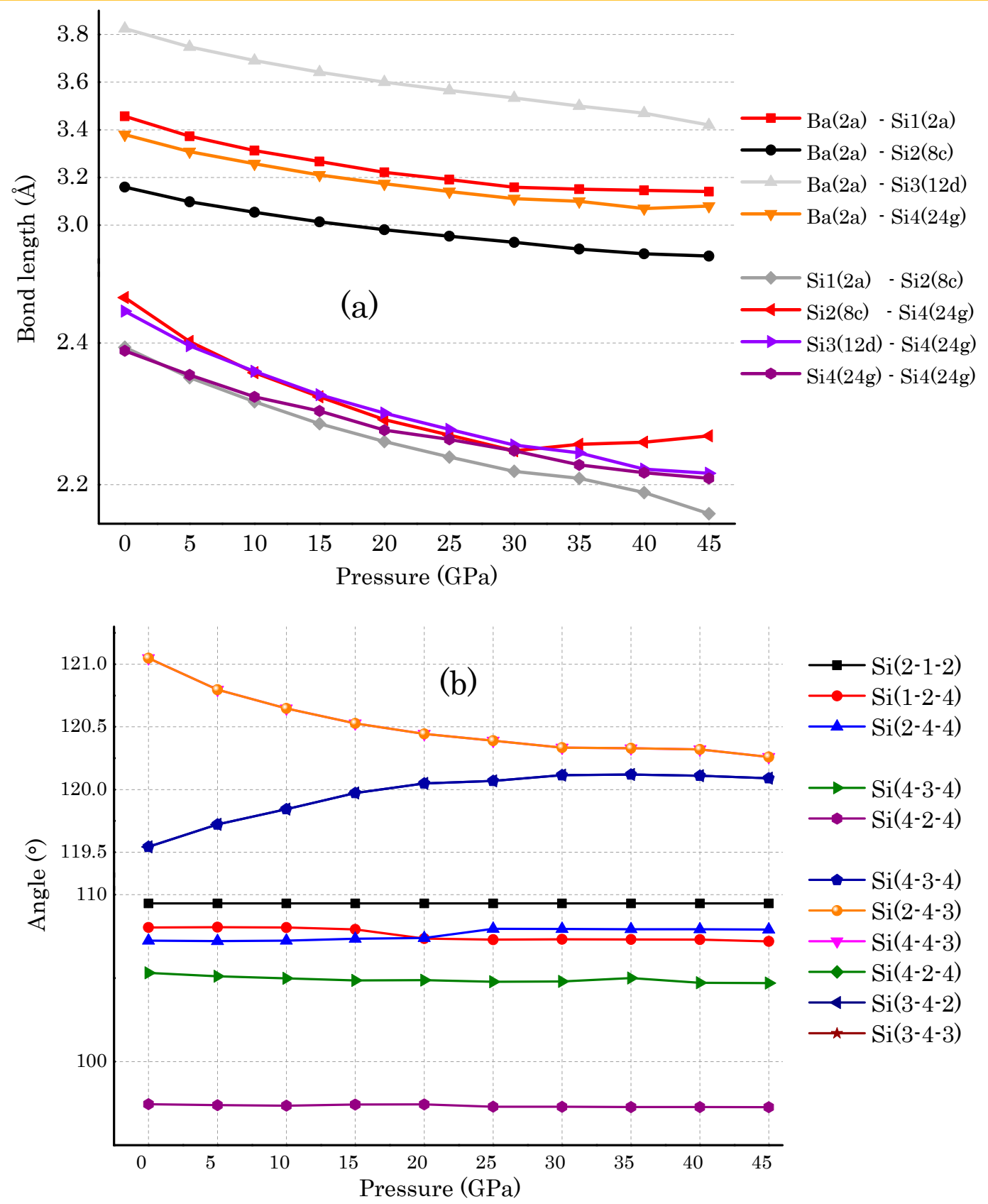

Figure 3. High-pressure behavior of: (a) Ba-Si and Si-Si distances, and (b) the Si-Si-Si angles for the type-VIII $\mathrm{Ba}_{8} \mathrm{Si}_{46}$ 
Peer-reviewed version available at Silicon 2019, 12; doi:10.1007/s12633-019-00145-1

Table.1: Optimized lattice parameters, atomic positions (Wyckoff notation), formation energy and mass density of the type-VIII $\mathrm{Ba}_{8} \mathrm{Si}_{46}$ silicon clathrate (space group Im (223)) using the GGA-PBE approximation within the CASTEP package.

\begin{tabular}{|c|c|c|c|c|}
\hline \multicolumn{5}{|c|}{ Lattice constant $a_{0}(\AA)$} \\
\hline \multicolumn{2}{|l|}{$10.395^{\mathrm{a}}$} & $10.385^{\mathrm{b}}$ & & $10.38^{c}$ \\
\hline Atom & Site: & $\mathrm{X}$ & $\mathrm{Y}$ & Z \\
\hline $\operatorname{Sil}(2 a)^{a}$ & $(x, y, z)$ & 0 & 0 & 0 \\
\hline $\operatorname{Si} 2(8 c)^{a}$ & $(x=y=z)$ & 0.367137 & 0.367137 & 0.367137 \\
\hline $\operatorname{Si3}(12 d)^{a}$ & $(x, y, z)$ & 0.25 & 0.5 & 0 \\
\hline $\mathrm{Si} 4(24 \mathrm{~g})^{\mathrm{a}}$ & $(x=y=z)$ & 0.418912 & 0.418912 & 0.418912 \\
\hline $\mathrm{Ba}(8 \mathrm{c})^{\mathrm{a}}$ & $(x=y=z)$ & 0.191830 & 0.191830 & 0.191830 \\
\hline \multicolumn{5}{|c|}{ Formation energy $\left(\mathrm{kJ} \mathrm{mol}^{-1}\right)$} \\
\hline \multicolumn{2}{|c|}{$-97.23^{\mathrm{a}}$} & $-97.17^{b}$ & \multicolumn{2}{|c|}{$-94.05^{\mathrm{c}}$} \\
\hline \multicolumn{5}{|c|}{ Density $\left(\mathrm{Kg} / \mathrm{m}^{3}\right)$} \\
\hline \multicolumn{2}{|c|}{$3.82 \times 10^{3 \mathrm{a}}$} & $3.7 \times 10^{3 b}$ & & \\
\hline \multicolumn{5}{|c|}{ Fundamental bandgap (eV) } \\
\hline \multicolumn{2}{|c|}{$1.07^{\mathrm{a}}$} & $1^{\mathrm{b}}$ & \multicolumn{2}{|c|}{$1^{\mathrm{c}}$} \\
\hline \multicolumn{5}{|c|}{$\begin{array}{l}\text { a: our work GGA-PBE } \\
b: \text { Ref [16] GGA-PBE } \\
c: \text { Ref [30] GGA-PBE }\end{array}$} \\
\hline
\end{tabular}

\section{III.2. Electronic properties}

\section{III.2.a. At zero pressure}

We have computed by means of GGA-PBE the band structures and the total and partial densities of states DOS of $\mathrm{Ba}_{8} \mathrm{Si}_{46}$-VIII under zero and high-pressure conditions. According to the space group (I4-3m, $\mathrm{N}^{\circ} 217$ ), the band structures are calculated along the following path at the first Brillouin zone: $\Gamma(0,0,0), \mathrm{H}(1 / 2,2 / 1,-1 / 2), \mathrm{N}(1 / 2,0,0), \mathrm{P}(1 / 4,1 / 4,1 / 4)$. Figure 4(a) illustrates the band structure and the figure 4(b) the partial DOS at 0GPa. The Bas $\mathrm{Si}_{46}-\mathrm{VIII}$ clathrate has a similar allure of band structure with the guest-free counterpart, they differ by the position of the Fermi level, generally, inorganic group-IV clathrates obey the rigid band model [38], according to which they will qualitatively have similar allures of band structures regardless the nature and the size of the intercalated species as long as the host framework atoms remain the same. This is due to the fact that guest atoms will contribute with their valence electrons to the framework's antibonding states, and hence, the Fermi level will be shifted towards the conduction band, creating mostly n-type heavily or lightly doped semiconducting materials (upon the concentration of guest atoms). Valance and conduction bands are not overlapped at the Fermi level (set to $0 \mathrm{eV}$ as energy reference). This result is coherent with previous studies by Norouzzadeh et al [16, 30], who has shown that $\mathrm{Ba}_{8} \mathrm{Si}_{46}-\mathrm{VIII}$ is a metallic-like compound with a fundamental bandgap of about $1 \mathrm{eV}$ (GGA-PBE) appearing between $\Gamma$ and $\mathrm{N}$ high-symmetry points. We found that the fundamental bandgap between the two same points is about $1.07 \mathrm{eV}$. 
Peer-reviewed version available at Silicon 2019, 12; doi:10.1007/s12633-019-00145-1

The total and partial densities of states DOS are illustrated in the figure 4(b). Structural stability of a compound is directly associated with its bonding strength. It can be noticed that near the valence band, the $\mathrm{Si}$ atoms contribute with their p-states, while the contribution from Si-s states increases when going toward low energies. Ba atoms contribute through their $s$ and $p$ states mainly to the middle and lower parts of the valence band, and they are less present in the conduction band.

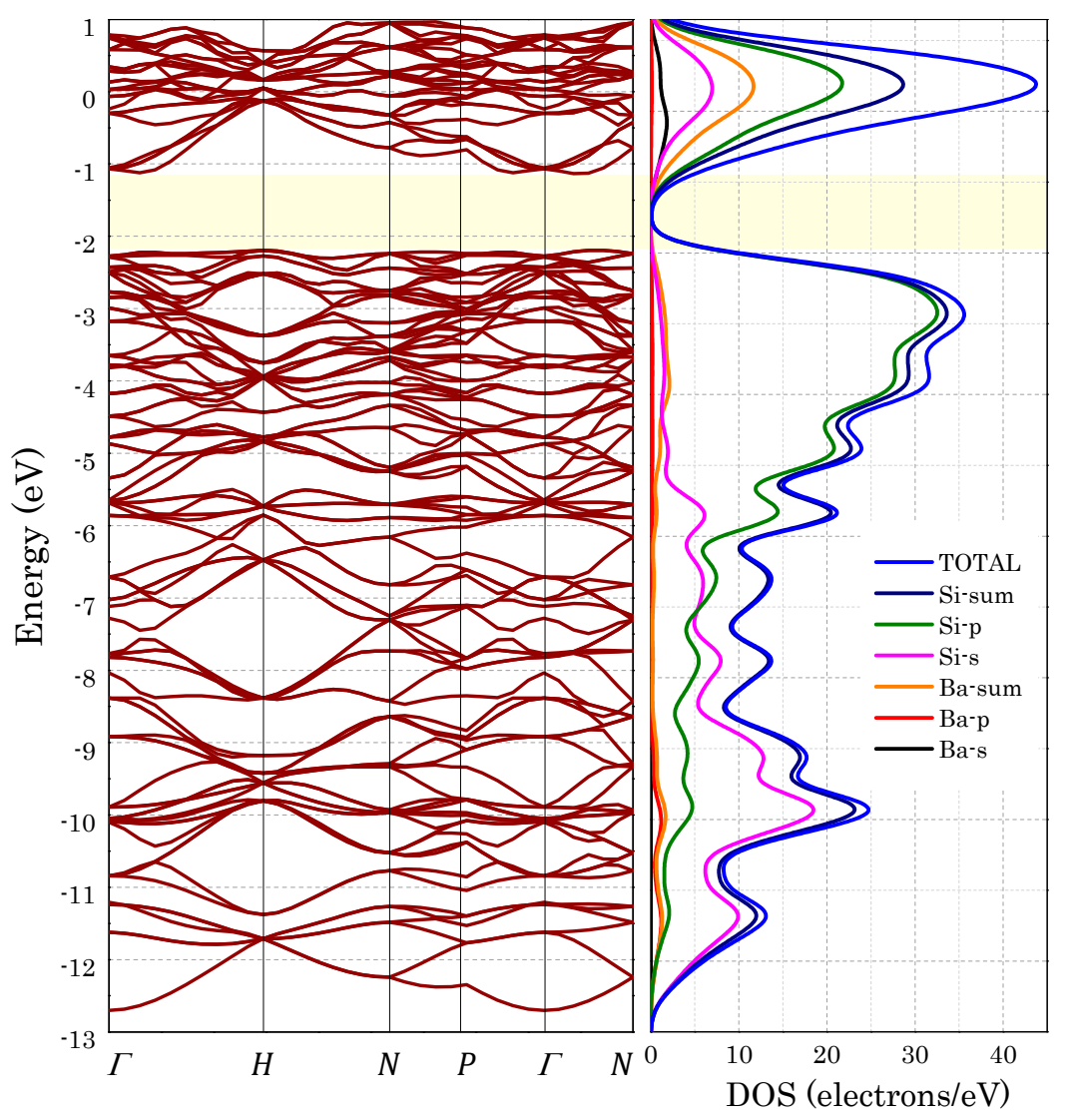

Figure 4. Electronic properties of VIII-Bas $\mathrm{Si}_{46}$ : (a) the band structure, and (b) the total and partial DOS.

\section{III.2.b Under pressure}

In order to study the impact of hydrostatic pressure on the electronic properties, we compare the band structures and densities of states (DOS) of type-VIII BasSi46 under 0, 15, and $30 \mathrm{GPa}$. In Fig. 5 are displayed the GGA-PBE band structures of the $\mathrm{Ba}_{8} \mathrm{Si}_{46}-\mathrm{VIII}$ along the $\Gamma$-H-N-P highsymmetry directions for (a) $0 \mathrm{GPa}$ (b) $15 \mathrm{GPa}$ and (c) $30 \mathrm{GPa}$. The Fermi level is set to $0 \mathrm{eV}$ as an energy reference. It can be noticed that within the fundamental bandgap, the top of the valence band (VBM) is located at the $\mathrm{N} k$-point and remains at its position under hydrostatic pressure, whereas, the conduction band minimum is located at $\Gamma-\mathrm{H}$ for $0 \mathrm{GPa}$ and $15 \mathrm{GPa}$ and moves to $\Gamma$ under $30 \mathrm{GPa}$. At $\Gamma$, higher alignment with other valleys is observed at the $\mathrm{CB}$ edge, 
Peer-reviewed version available at Silicon 2019, 12; doi:10.1007/s12633-019-00145-1

and a smaller effective mass at this point is estimated which can lead to higher electronic mobility [16], and therefore to better semiconducting and thermoelectric properties. The fundamental band gap slightly increases under pressure; it goes from $1.07 \mathrm{eV}$ at $0 \mathrm{GPa}$ to 1.12 $\mathrm{eV}$ at $15 \mathrm{GPa}$, and to $1.17 \mathrm{eV}$ under $30 \mathrm{GPa}$. This can be explained in terms of partial density of states. In Figure 6, contributions from $\mathrm{Ba}$ and $\mathrm{Si}$ states become stronger under high pressure, Due to the strong hybridization of the Si-s and Ba-p states at Fermi level at higher pressures; at 30 GPa we observe an additional peak of the Ba-p states at $15 \mathrm{eV}$ energy level. Also, the contribution from silicon states near and beyond the Fermi level increases with increasing pressure.
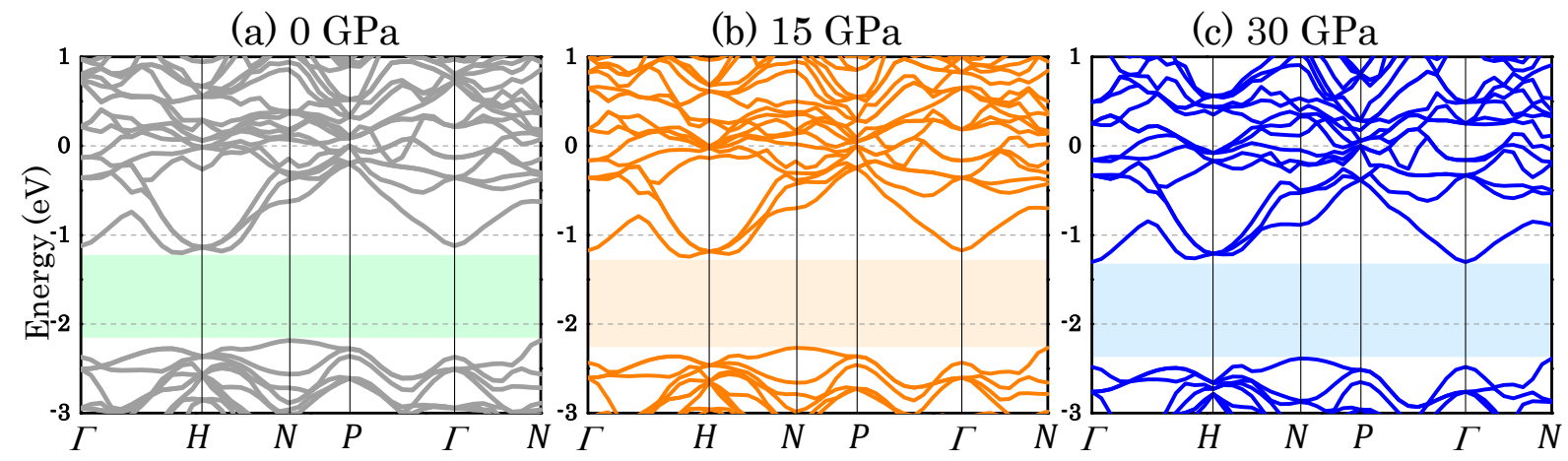

Figure 5. High-pressure effect on the band structure of the type-VIII Ba8 $\mathrm{Si}_{46}$ at: (a) 0GPa, (b) 15 $\mathrm{GPa}$, and (c) $30 \mathrm{GPa}$.

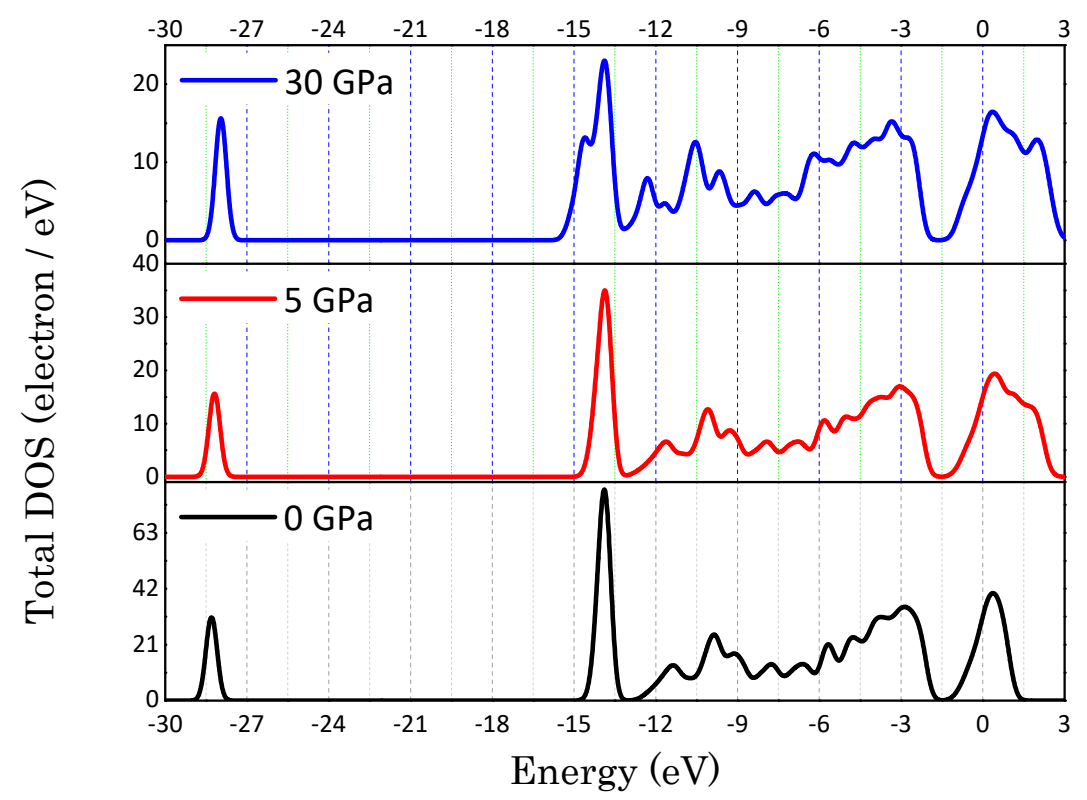

Figure 6. Influence of high-pressure on the total density of states DOS of the type-VIII Bar $\mathrm{Si}_{46}$. 


\section{III.3. Elastic constants and modulus}

\section{III.3.a. At zero pressure}

The type-VIII $\mathrm{Ba}_{8} \mathrm{Si}_{46}$ clathrate crystallize into a body centered cubic structure; therefore, only three independent elastic constants $\left(C_{11}, C_{12}\right.$ and $\left.C_{44}\right)$ are sufficient to describe the elastic behavior of the material. Defining these elastic constants and investigating their behavior under high-pressure is primordial to predict the mechanical stability of the investigated material under such conditions during synthesis. For sake of comparison, elastic constants and modulus at zero pressure were also computed for the type-VIII guest-free $\mathrm{Si}_{46}$, and for the type-I $\mathrm{Si}_{46}$ and $\mathrm{Ba}_{8} \mathrm{Si}_{46}$ by means of the same GGA-PBE approach and settings. Results are compared with the available literature. The total energy of a given symmetrical crystal at zero pressure and at its equilibrium volume state $V_{0}$ is given by:

$\Delta E(\varepsilon)=\frac{1}{2} V_{0} C_{i j} \varepsilon^{2}+0\left[\varepsilon^{4}\right]$

Where: $\Delta E(\varepsilon)=E(\varepsilon)-E_{0}$ is the total energy difference. $C_{i j}$ are the elastic constants and $\varepsilon$ are the strain tensor elements. According to the Hooke's law of general elasticity $\sigma_{i j}=C_{i j k l} \varepsilon_{k l}$, elastic constants can be calculated through the second derivatives of the total energy. Moreover, the three elastic constants are generally derived from a set of three equations. The resolution of the first two equations, which implicate the bulk modulus $\mathrm{B}$ and the shear constant $\mathrm{C}^{\prime}$, will give $\mathrm{C}_{11}$ and $\mathrm{C}_{12}$ as described hereafter.

$$
\begin{aligned}
& B=\frac{1}{3}\left(C_{11}+2 C_{12}\right) \\
& C^{\prime}=\frac{1}{2}\left(C_{11}-C_{12}\right)
\end{aligned}
$$

The bulk modulus $B$ is obtained by applying a hydrostatic strain tensor and then by fitting the Munaghan equation of state. The shear constant $C^{\prime}$ is obtained through applying a volumeconserving tetragonal stress tensor:

$$
\mathrm{r} \varepsilon=\left(\begin{array}{ccc}
\varepsilon & 0 & 0 \\
0 & \varepsilon & 0 \\
0 & 0 & \frac{1}{(1+\varepsilon)^{2}}-1
\end{array}\right)
$$

Application of such tensor will change the total energy as follows:

$$
\Delta E(\varepsilon)=6 V_{0} C^{\prime} \varepsilon^{2}+0\left[\varepsilon^{4}\right]
$$


Peer-reviewed version available at Silicon 2019, 12; doi:10.1007/s12633-019-00145-1

The third equation gives the $\mathrm{C}_{44}$ constant through applying an orthorhombic stress tensor

$$
\varepsilon=\left(\begin{array}{ccc}
0 & \varepsilon & 0 \\
\varepsilon & 0 & 0 \\
0 & 0 & \frac{\varepsilon^{2}}{1-\varepsilon^{2}}
\end{array}\right)
$$

This total energy is obtained as:

$$
\Delta E(\varepsilon)=2 V_{0} C_{44} \varepsilon^{2}+0\left[\varepsilon^{4}\right]
$$

Then the shear modulus $\mathrm{G}$ is given for a cubic system as:

$$
G=\frac{1}{5}\left(C_{11}-C_{12}+3 C_{44}\right)
$$

The Young's modulus E is given as:

$$
E=\frac{9 B G}{3 B+G}
$$

Additionally, the Young's modulus E along $<100>$ and $<111>$ directions are respectively described as:

$$
\begin{aligned}
& E_{(100)}=\frac{\left(C_{11}+2 C_{12}\right)\left(C_{11}-C_{12}\right)}{C_{11}+C_{12}} \\
& E_{(111)}=\frac{1}{E} \frac{C_{11}-C_{12}-2 C_{44}}{3 C_{44}\left(C_{11}+C_{12}\right)}
\end{aligned}
$$

The average speed of sound $v$ in a material is given as [39]:

$$
v=\left[\frac{1}{3}\left(\frac{2}{v_{\text {tran }}^{3}}+\frac{1}{v_{\text {long }}{ }^{3}}\right)\right]^{-1 / 3}
$$

Where vlong and $v_{\text {tran }}$ are the longitudinal and transversal sound velocities, which are expressed as:

$$
v_{\text {long }}=\sqrt{\left(\frac{3 B+4 G}{3 \rho}\right)} \text { and } v_{\text {tran }}=\sqrt{\left(\frac{G}{\rho}\right)}
$$

Where: $\rho$ represents the mass density $\left(\mathrm{Kg} / \mathrm{m}^{3}\right)$.

First, we examine the elastic properties of the Ba8Si46-VIII at zero pressure. The numerical values of the above-described elastic constants are estimated using the CASTEP code by applying a small set of deformations to the crystal and taking the second derivatives of the total energy as expressed in equation (1). Our results of elastic constants $C i j$, the bulk modulus $B$, the shear modulus $G$, the Young's modulus $E$, and the average velocity of sound $v$, calculated by GGA-PBE for $\mathrm{Ba}_{8} \mathrm{Si}_{46}$-VIII at $0 \mathrm{GPa}$ are listed in table 2, and compared with reported data for type-I and type-VIII Si clathrates. Our results are in a fair agreement with the literature. We 
notice from table 2 that VIII-Ba8Si46 having a bulk modulus of $\mathrm{B}=75.804 \mathrm{GPa}$, lower than that of the type-I Bas $\mathrm{Si}_{46}(\mathrm{~B}=76 \mathrm{GPa})$, and that of the guest-free $\mathrm{Si}_{46}$ type-I (B=76.92 GPa) and typeVIII $(\mathrm{B}=78.87 \mathrm{GPa})$. The bulk modulus $\mathrm{B}$, which presents a measure of resistance of the material against external deformation, provides much information about both the hardness and the bonding strength of the studied material. Our results confirm that the type-VIII Ba8 $\mathrm{Si}_{46}$ is less resistant to external deformation than its counterparts. However, resistance to plastic deformation is also proportional to the elastic shear modulus $G$. Additionally, the shear modulus $G$, the Young's modulus $E$, and the average velocity of sound $v$ are all larger for the type-VIII Ba8Si46 than those for the type-I Bas $\mathrm{Si}_{46}$ but reduced when compared with guest-free counterparts $\mathrm{Si}_{46}$. Ductility and fragility play a critical role during the synthesis of materials. In general, a ductile material is able to deform a lot before it is broken unlike a fragile material which breaks without or with less deformation, we can therefore predict if the material is fragile or ductile upon the well-known Pugh ratio $G / B$ [40], if this ratio is equal or lower than 1.74 the material is least malleable (fragile), otherwise it is more malleable (ductile). In our case we predict that $B / G=$ 1.681, slightly lower than the given value, this indicates that the material should be fragile at zero pressure. Elastic constants $C_{11}, C_{12}$ and $C_{44}$ are slightly reduced for the type-VIII Ba8Si46. The first elastic constant $C_{11}$, which quantitatively represents the uniaxial deformation along the $<001>$ direction has the highest value, indicating the incompressible character of the compound under the $<001>$ uniaxial stress and is relatively smaller in type-I than in the type-VIII Si46. One can approximately define the melting temperature $\mathrm{T}_{\mathrm{m}}$ of a cubic crystal from $C_{11}$ through the equation suggested by Fine et al [41] where $\mathrm{T}_{\mathrm{m}}=553+\left(5.91 \mathrm{C}_{11}\right) \pm 300 \mathrm{~K}\left(\mathrm{~T}_{\mathrm{m}}\right.$ in $\mathrm{K}, \mathrm{C}_{11}$ in $\left.\mathrm{GPa}\right)$, we therefore predict for the type-VIII $\mathrm{Ba}_{8} \mathrm{Si}_{46}$ that $\mathrm{T}_{\mathrm{m}}=1274.634 \mathrm{~K} \pm 300 \mathrm{~K}$. Considering the Born's stability criteria for cubic crystals [42] $\mathrm{C}_{11}>0, \mathrm{C}_{11}-\mathrm{C}_{12}>0, \mathrm{C}_{11}+2 \mathrm{C}_{12}>0$ and $\mathrm{C}_{44}>0$, the two types are mechanically stable. The Si46-VIII seems to be harder than its type-I counterpart. Our results are in good agreement with those reported by Norouzzadeh et al [17], who predicted that $\mathrm{Si}_{46}$-VIII is a strongly-isotropic material, and stable against shear and high-pressure, similarly to the type-I Si46. However, empty clathrates, in general, tend to have lower bulk modulus than the guest-containing clathrates, the guest-host hybridization usually plays an important role that can lead to hard 'diamond-like' materials [10]. But in contrary, our result shows that intercalation of $8 \mathrm{Ba}$ atoms into the Si46 framework reduces the bulk modulus by about $17 \%$, this is in fact due to the expansion of the Si cages caused by the presence of big $\mathrm{Ba}$ atoms and therefore the weakening effect of this doping over the overall hardness of the material. Furthermore, it was demonstrated through many experimental and theoretical reports that the presence of guest atoms might decrease the stability of clathrates through these host-guest interactions that weakens the 
Peer-reviewed version available at Silicon 2019, 12; doi:10.1007/s12633-019-00145-1

Si-Si bonding on one hand [43], and in the other hand, the electron exchange enforces the integrity of the clathrates frameworks [37]. The main challenge for obtaining a guest-free clathrate $\mathrm{Si}_{46}$, whether in type-I or VIII phases, is the fact that removing the guest atoms from the cages will induce a collapse of the system and hence a phase transformation towards more stable phases such as diamond structure $\mathrm{Si}_{2}[10,37]$. Nevertheless, guest-free type-II Si136 clathrate, was the only guest-free silicon clathrate to be successfully synthesized as crystalline [13], or thin film forms [15].

\section{III.3.b. Under high-pressure}

In order to probe the impact of hydrostatic pressure on the mechanical behavior of VIII-Ba8 $\mathrm{Si}_{46}$, we have calculated (using the same GGA-PBE functional with the same computational procedures as precedent calculations) the elastic constants and modulus for optimized structures under high-pressure (from $0 \mathrm{GPa}$ to $45 \mathrm{GPa}$ ). Figure 7(a) illustrates the evolution of calculated elastic constants $\mathrm{C}_{\mathrm{ij}}$ under high-pressure, unfortunately, comparison with other experimental or theoretical data is not possible since such studies are not yet reported. It can be noticed that $\mathrm{C}_{11}$ and $\mathrm{C}_{12}$ have similar monotonous pressure dependence behaviors, however their magnitudes are quite different, whereas, $\mathrm{C}_{44}$ increases slowly and is less affected by pressure. The $\mathrm{C}_{44}$ variation with pressure shows that a maximum value of $55.72 \mathrm{GPa}$ is reached at $10 \mathrm{GPa}$, and then it tends to stabilize under higher values of pressure. Considering the previously pointed stability criterion for cubic crystals, the $\mathrm{C}_{\mathrm{ij}}$ constants remain all positive under pressure. However, the previous criterion does not provide alone a precise prediction of the high-pressure stability of the material in question, therefore, the following conditions will be applied and the results are checked afterwards, and if fulfilled, the cubic material might be stable under such isotropic-pressure conditions [44]:

$\tilde{C}_{44}>0, \quad \tilde{C}_{11}>\left|\tilde{C}_{12}\right|, \quad \tilde{C}_{11}+2 \tilde{C}_{12}>0$

Where $\tilde{C}_{11}, \tilde{C}_{12}$ and $\tilde{C}_{44}$ present the elastic constants under pressure, and are given as:

$\tilde{C}_{11}=C_{11}-P, \quad \tilde{C}_{12}=C_{12}+P, \quad \tilde{C}_{44}=C_{44}-P$

$\mathrm{P}$ is the pressure in $\mathrm{GPa}$.

Figure $7\left(\right.$ b) illustrates the evolution of $\tilde{C}_{11}, \tilde{C}_{12}$ and $\tilde{C}_{44}$ along with the third criterion $\tilde{C}_{11}-\tilde{C}_{12}$ that is equal to: $C_{11}-C_{12}-2 P$ and which presents the precedent stability criterion under highpressure. When pressure increases, our calculations show that the values of $C_{11}-C_{12}-2 P$ will not remain positive under all the pressure range, and the conditions are not fulfilled though, 
Peer-reviewed version available at Silicon 2019, 12; doi:10.1007/s12633-019-00145-1

therefore, we predict that type-VIII Ba8Si46 will not be stable for pressures above $24 \mathrm{GPa}$. Henceforward, the compound VIII-Bar $\mathrm{Si}_{46}$ is expected to remain mechanically stable during and after synthesis below $24 \mathrm{GPa}$, however, we have to clarify that this criterion is not sufficient alone to predict any phase transition that can occur below $24 \mathrm{GPa}$, which is needed to be proven through experiments.
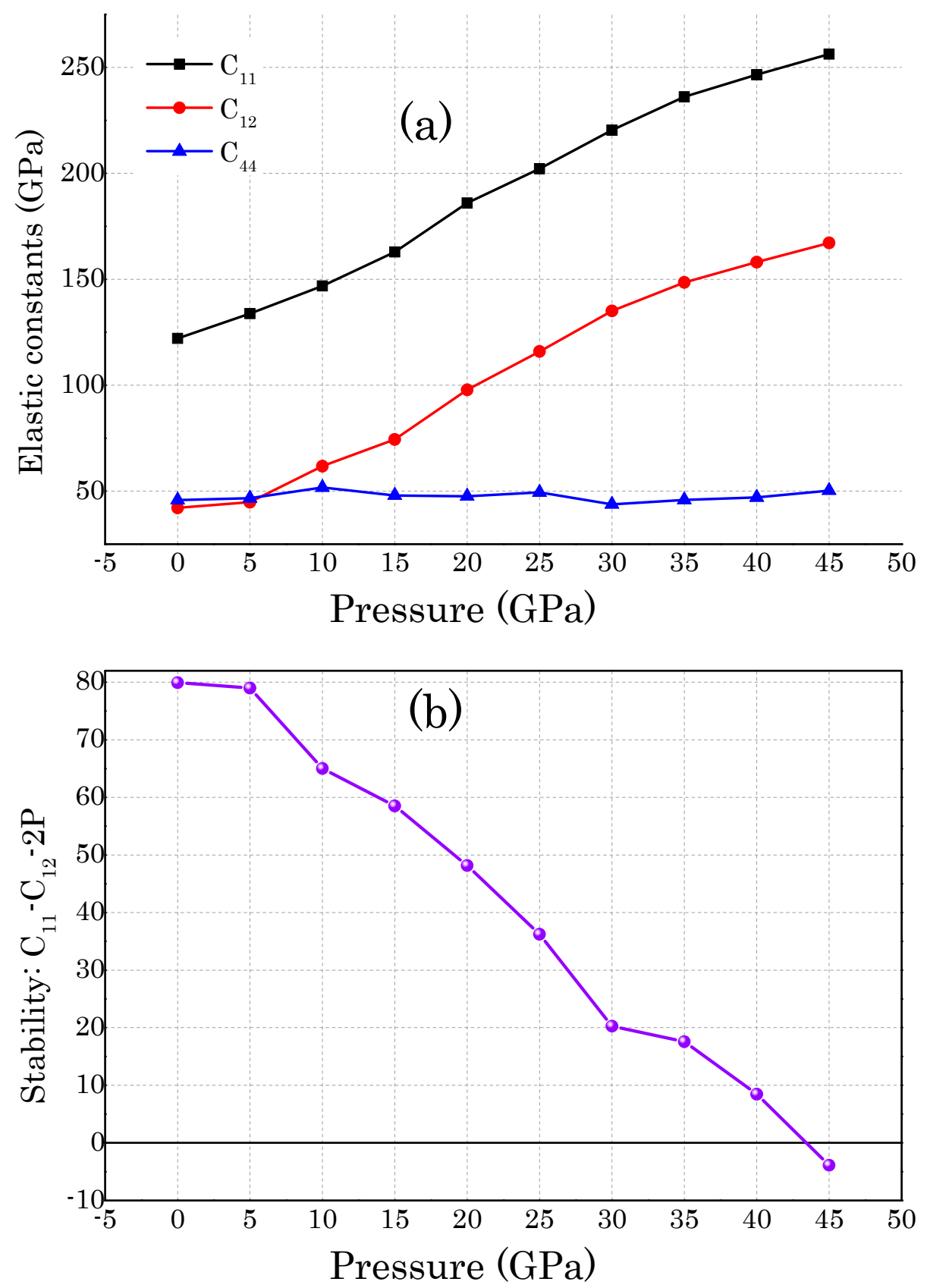

Figure 7. High-pressure evolution of (a) elastic constants $C_{11}, C_{12}$ and $C_{44}$, and (b) the stability criterion $C_{11}-C_{12}-2 P$ for the type-VIII $\mathrm{Ba}_{8} \mathrm{Si}_{46}$ 
Peer-reviewed version available at Silicon 2019, 12; doi:10.1007/s12633-019-00145-1

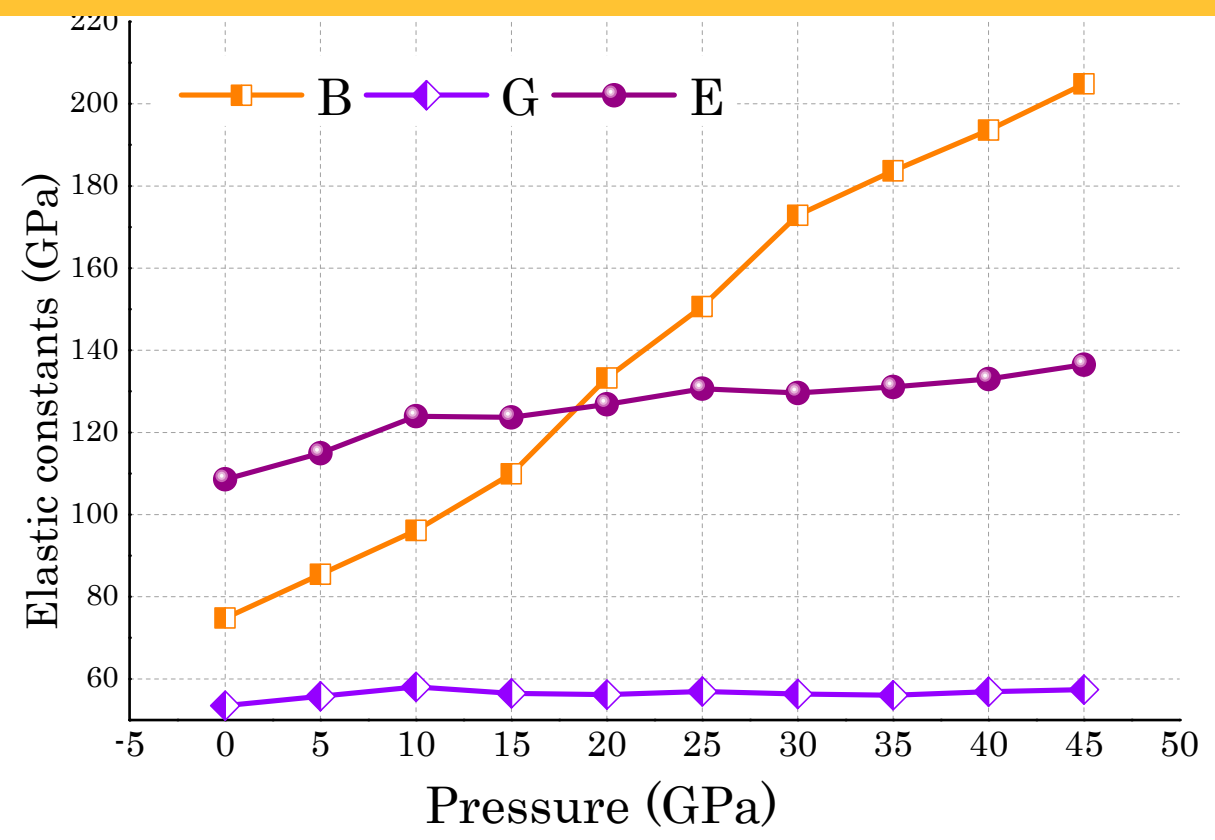

Figure 8. High-pressure evolution of major elastic modulus including the bulk modulus $B$, the shear modulus $G$ and the Young's modulus $E$, for the type-VIII Bas $\mathrm{Si}_{46}$

The major elastic modulus of VIII-Bas $\mathrm{Si}_{46}$ under high pressure are computed and displayed in figure 8 . It can be observed that the bulk modulus $B$ increments monotonously with pressure in a linear manner, this was expected and is due to the fact that $B$ is proportional to the applied pressure and inversely proportional to the volume change through the common physical equation of the bulk modulus $(\mathrm{B}=\Delta \mathrm{P} / \Delta \mathrm{V})$. Young's modulus $E$ which represents the resistance of the material to uniaxial strains and provides the strength degree is found to rapidly increase from 0 to $10 \mathrm{GPa}$ and then starts to slowly increase beyond that pressure, this indicated the incremented stiffness of the material towards pressure. The shear modulus $G$ which describes the resistance towards shearing forces is less affected by pressure, but it tends to slightly increase and stabilizes after $10 \mathrm{GPa}$ at about $56.50 \mathrm{GPa}$. The values of $E$ and $G$ both have similar behaviors towards pressure, even slowly, this means that, the capacity of the resist stiffness and shear deformation of type-VIII Bas $\mathrm{Si}_{46}$ are increased with the increase of pressure respectively. However, the typeVIII $\mathrm{Ba}_{8} \mathrm{Si} 46$ is globally more resistive to volume compressions than to shear deformation since in all cases $\mathrm{B}>G$ for the pressure range. 


\begin{tabular}{|c|c|c|c|c|}
\hline \multicolumn{3}{|c|}{ 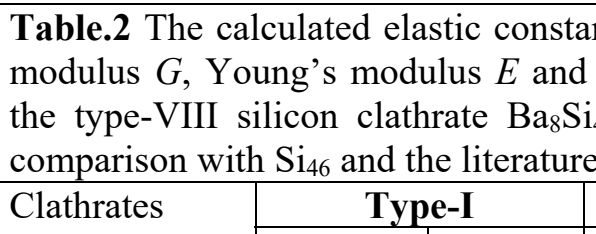 } & \multicolumn{2}{|c|}{ Type-VIII } \\
\hline & $\mathrm{Si}_{46}$ & $\mathrm{Ba}_{8} \mathrm{Si}_{46}$ & $\mathrm{Si}_{46}$ & $\mathrm{Ba}_{8} \mathrm{Si}_{46}$ \\
\hline$C_{11}(\mathrm{GPa})$ & $\begin{array}{l}134.89^{\mathrm{a}} \\
140^{\mathrm{b}}\end{array}$ & $135^{\mathrm{f}}$ & $\begin{array}{l}146.17^{\mathrm{a}} \\
149.28^{\mathrm{b}} \\
139.3^{\mathrm{c}}\end{array}$ & $\begin{array}{l}122.104^{\mathrm{a}} \\
125.46^{\mathrm{d}}\end{array}$ \\
\hline$C_{12}(\mathrm{GPa})$ & $\begin{array}{l}47.93^{\mathrm{a}} \\
47^{\mathrm{b}}\end{array}$ & $46.3^{\mathrm{f}}$ & $\begin{array}{l}45.22^{a} \\
49.22^{b} \\
46.3^{c}\end{array}$ & $\begin{array}{l}42.154^{\mathrm{a}} \\
43.26^{\mathrm{d}}\end{array}$ \\
\hline$C_{44}(\mathrm{GPa})$ & $\begin{array}{l}47.8^{\mathrm{a}} \\
47^{\mathrm{b}}\end{array}$ & $24.7^{\mathrm{f}}$ & $\begin{array}{l}45.88^{a} \\
46.83^{b} \\
42.3^{c}\end{array}$ & $\begin{array}{l}45.825^{\mathrm{a}} \\
46.5^{\mathrm{d}}\end{array}$ \\
\hline$B(\mathrm{GPa})$ & $\begin{array}{l}76.92^{\mathrm{a}} \\
78^{\mathrm{b}} \\
78.5^{\mathrm{c}} \\
87^{\mathrm{d}}\end{array}$ & $\begin{array}{l}76^{f} \\
93^{g}\end{array}$ & $\begin{array}{l}78.87^{\mathrm{a}} \\
82.57^{\mathrm{b}} \\
80.3^{\mathrm{c}}\end{array}$ & $\begin{array}{l}75.804^{\mathrm{a}} \\
76.66^{\mathrm{d}}\end{array}$ \\
\hline$G(\mathrm{GPa})$ & $\begin{array}{l}46.07^{\mathrm{a}} \\
46^{\mathrm{b}}\end{array}$ & $32.6^{\mathrm{f}}$ & $\begin{array}{l}47.72^{\mathrm{a}} \\
48.08^{\mathrm{b}}\end{array}$ & $\begin{array}{l}45.085^{\mathrm{a}} \\
45.17^{\mathrm{d}}\end{array}$ \\
\hline$E(\mathrm{GPa})$ & $\begin{array}{l}115.21^{\mathrm{a}} \\
115.33^{\mathrm{b}}\end{array}$ & $85.6^{\mathrm{f}}$ & $\begin{array}{l}119.133^{\mathrm{a}} \\
120.80^{\mathrm{b}}\end{array}$ & $\begin{array}{l}108.436^{\mathrm{a}} \\
109.57^{\mathrm{d}}\end{array}$ \\
\hline$v(\mathrm{~m} / \mathrm{s})$ & $\begin{array}{l}4402^{\mathrm{a}} \\
4931^{\mathrm{b}} \\
5345^{\mathrm{h}}\end{array}$ & $3390^{f}$ & $\begin{array}{l}5261.7^{\mathrm{a}} \\
5196^{\mathrm{b}} \\
5053^{\mathrm{h}}\end{array}$ & $\begin{array}{l}4695^{\mathrm{a}} \\
4509^{\mathrm{d}}\end{array}$ \\
\hline \multicolumn{5}{|c|}{$\begin{array}{l}\text { a: This work GGA-PBE (CASTEP) } \\
\text { b: data from ref [17] GGA-PBE (ABINIT) } \\
\text { c: data from ref [45] GGA-PBE (QUANTUM-ESPRESSO) } \\
\text { d: data from ref [30] } \\
\text { e: data from ref [46] LDA (SIESTA) } \\
\text { f: data from ref [47] Hartree-Fock HF (CRYSTAL 95) } \\
\text { g: data from ref [36] } \\
\text { h: data from ref [34] } \\
\text { i: data from ref [48] }\end{array}$} \\
\hline
\end{tabular}

\section{Conclusion}

In this paper, first-principles calculations in the frame of the Density Functional theory DFT were solicited to investigate the high-pressure behavior of the structural, electronic and mechanical properties of the type-VIII Ba ${ }_{8} \mathrm{Si}_{46}$ silicon clathrate. The structural evolution shows a stable behavior with pressure with no significant indices of possible phase transition or volume collapse. Electronic properties are highly affected by the presence of pressure, the metallic character of the compound is not changed throughout the pressure range, however, the interaction between barium atoms and their surrounding Si environment becomes stronger with pressure, and as a consequence, the Fermi level decreases. Elastic properties are also affected by pressure, the major mechanical properties were computed for relaxed and stressed materials, and our results showed that the type-VIII $\mathrm{Ba}_{8} \mathrm{Si}_{46}$ is relatively hard and resistant against deformation 
and shear forces and might collapse if pressure exceeds $24 \mathrm{GPa}$, however, our predictions are to be confirmed through experiments.

\section{References}

1. Kasper, J.S., et al., Clathrate structure of silicon Na8Si46 and NaxSi136 ( $x<11)$. Science, 1965. 150(3704): p. 1713-1714.

2. Cros, C., M. Pouchard, and P. Hagenmuller, Sur une nouvelle famille de clathrates minéraux isotypes des hydrates de gaz et de liquides. Interprétation des résultats obtenus. Journal of Solid State Chemistry, 1970. 2(4): p. 570-581.

3. Bentien, A., et al., Thermal conductivity of thermoelectric clathrates. Physical Review B, 2004. 69(4).

4. Dolyniuk, J.-A., et al., Clathrate thermoelectrics. Materials Science and Engineering: R: Reports, 2016. 108: p. 1-46.

5. Martinez, A.D., et al., Synthesis of Group IV Clathrates for Photovoltaics. Photovoltaics, IEEE Journal of, 2013. 3(4): p. 1305-1310.

6. Connétable, D. and X. Blase, Electronic and superconducting properties of silicon and carbon clathrates. Applied Surface Science, 2004. 226(1-3): p. 289-297.

7. Connétable, D., et al., Superconductivity in Dopedsp3Semiconductors: The Case of the Clathrates. Physical Review Letters, 2003. 91(24).

8. Yang, J. and J.S. Tse, Silicon clathrates as anode materials for lithium ion batteries? Journal of Materials Chemistry A, 2013. 1(26): p. 7782.

9. Yamanaka, S., Silicon clathrates and carbon analogs: high pressure synthesis, structure, and superconductivity. Dalton Trans, 2010. 39(8): p. 1901-15.

10. San-Miguel, A. and P. Toulemonde, High-pressure properties of group IV clathrates. High Pressure Research, 2005. 25(3): p. 159-185.

11. Jonathan W. Steed, D.R.T., Karl J. Wallace, Core concepts in supramolecular chemistry and nanochemistry2007.

12. Powell, H.M., 15. The structure of molecular compounds. Part IV. Clathrate compounds. Journal of the Chemical Society (Resumed), 1948(0): p. 61.

13. Gryko, J., et al., Low-density framework form of crystalline silicon with a wide optical band gap. Physical Review B, 2000. 62(12): p. R7707-R7710.

14. Nolas, G.S., et al., Raman scattering study of stoichiometric Si and Ge type II clathrates. Journal of Applied Physics, 2002. 92(12): p. 7225-7230.

15. Kume, T., et al., Thin film of guest-free type-II silicon clathrate on Si(111) wafer. Thin Solid Films, 2016. 609: p. 30-34.

16. Norouzzadeh, P., et al., Type VIII Si based clathrates: prospects for a giant thermoelectric power factor. Physical Chemistry Chemical Physics, 2015. 17(14): p. 8850-8859.

17. Norouzzadeh, P., C.W. Myles, and D. Vashaee, Prediction of a large number of electron pockets near the band edges in type-VIII clathrate Si46 and its physical properties from first principles. J Phys Condens Matter, 2013. 25(47): p. 475502.

18. Norouzzadeh, P., C.W. Myles, and D. Vashaee, Prediction of giant thermoelectric power factor in type-VIII clathrate Si46. Sci Rep, 2014. 4: p. 7028.

19. Hohenberg, P. and W. Kohn, Inhomogeneous Electron Gas. Physical Review, 1964. 136(3B): p. B864-B871.

20. Kohn, W. and L.J. Sham, Self-Consistent Equations Including Exchange and Correlation Effects. Physical Review, 1965. 140(4A): p. A1133-A1138.

21. Clark, S.J., et al., First principles methods using CASTEP. Zeitschrift für Kristallographie, 2005. 220(5/6/2005). 
22. Hamann, D.R., M. Schlüter, and C. Chiang, Norm-Conserving Pseudopotentials. Physical Review Letters, 1979. 43(20): p. 1494-1497.

23. Perdew, J.P., K. Burke, and M. Ernzerhof, Generalized gradient approximation made simple. Physical Review Letters, 1996. 77(18): p. 3865.

24. Monkhorst, H.J. and J.D. Pack, Special points for Brillouin-zone integrations. Physical Review B, 1976. 13(12): p. 5188-5192.

25. Broyden, C.G., The convergence of a class of double-rank minimization algorithms 1. general considerations. IMA Journal of Applied Mathematics, 1970. 6(1): p. 76-90.

26. Fletcher, R., A new approach to variable metric algorithms. The computer journal, 1970. 13(3): p. 317-322.

27. Goldfarb, D., A family of variable-metric methods derived by variational means. Mathematics of computation, 1970. 24(109): p. 23-26.

28. Shanno, D.F., Conditioning of quasi-Newton methods for function minimization. Mathematics of computation, 1970. 24(111): p. 647-656.

29. Mahammedi, N.A., et al., Prediction of optically-active transitions in type-VIII guest-free silicon clathrate Si46: A comparative study of its physical properties with type-I counterpart through first-principles. Journal of Applied Physics, 2017. 122(20): p. 205103.

30. Norouzzadeh, P., C.W. Myles, and D. Vashaee, Structural, electronic, phonon and thermodynamic properties of hypothetical type-VIII clathrates Ba8Si46 and Ba8Al16Si30 investigated by first principles. Journal of Alloys and Compounds, 2014. 587: p. 474-480.

31. Shimizu, H., et al., Raman spectroscopy of type-I and type-VIII silicon clathrate alloys Sr8AlxGa16-xSi30. Journal of Alloys and Compounds, 2009. 487(1-2): p. 47-51.

32. Yamanaka, S., et al., High-Pressure Synthesis of a New Silicon Clathrate Superconductor, Ba8Si46. Inorg Chem, 2000. 39(1): p. 56-58.

33. Li, D.C., et al., Structural and electronic properties of type-I and type-VIII Ba8Ga16Sn30 clathrates under compression. Physica B: Condensed Matter, 2012. 407(8): p. 1238-1243.

34. Kim, D.Y. and T. Kume, Si allotropes and group IV clathrates investigated under high pressures. Japanese Journal of Applied Physics, 2017. 56(5S3): p. $05 F A 07$.

35. Leoni, S., W. Carrillo-Cabrera, and Y. Grin, Modelling of the $\alpha$ (clathrate VIII) $\rightleftharpoons 6$ (clathrate I) phase transition in Eu8Ga16Ge30. Journal of Alloys and Compounds, 2003. 350(1-2): p. 113122.

36. Zhang, W., et al., Influence of missing guest and host atoms on the mechanical and electronic properties of type-I clathrate compound Ba8Si46. Journal of Alloys and Compounds, 2015. 653: p. 77-87.

37. Tse, J.S., et al., Structural Stability and Phase Transitions inK8Si46Clathrate under High Pressure. Physical Review Letters, 2002. 89(19).

38. Nolas, G.S., The Physics and Chemistry of Inorganic Clathrates. Springer Series in Materials Science. Vol. 199. 2014: Springer Netherlands. 341.

39. Ghebouli, B., et al., Theoretical prediction of the structural, elastic, electronic, optical and thermal properties of the cubic perovskites CsXF3 (X $=\mathrm{Ca}, \mathrm{Sr}$ and $\mathrm{Hg}$ ) under pressure effect. Solid State Sciences, 2012. 14(7): p. 903-913.

40. Pugh, S.F., XCII. Relations between the elastic moduli and the plastic properties of polycrystalline pure metals. The London, Edinburgh, and Dublin Philosophical Magazine and Journal of Science, 1954. 45(367): p. 823-843.

41. Fine, M.E., L.D. Brown, and H.L. Marcus, Elastic constants versus melting temperature in metals. Scripta Metallurgica, 1984. 18(9): p. 951-956.

42. Born, M. and R. Oppenheimer, Zur Quantentheorie der Molekeln. Annalen der Physik, 1927. 389(20): p. 457-484.

43. Myles, C.W., J. Dong, and O.F. Sankey, Structural and electronic properties of tin clathrate materials. Physical Review B, 2001. 64(16). 
Peer-reviewed version available at Silicon 2019, 12; doi:10.1007/s12633-019-00145-1

44. Sin'ko, G.V. and N.A. Smirnov, Ab initio calculations of elastic constants and thermodynamic properties of bcc, fcc, and hcp Al crystals under pressure. Journal of Physics: Condensed Matter, 2002. 14(29): p. 6989.

45. Norouzzadeh, P. and C.W. Myles, A first-principles lattice dynamical study of type-I, type-II, and type-VIII silicon clathrates. Journal of Materials Science, 2016. 51(9): p. 4538-4548.

46. Connétable, D., et al., Tailoring Band Gap and Hardness by Intercalation: Anab initioStudy ofl8@Si-46and Related Doped Clathrates. Physical Review Letters, 2001. 87(20).

47. Perottoni, C.A. and J.A.H.d. Jornada, The carbon analogues of type-I silicon clathrates. Journal of Physics: Condensed Matter, 2001. 13(26): p. 5981.

48. Norouzzadeh, P., J.S. Krasinski, and T. Tadano, Thermal conductivity of type-I, type-Il, and type-VIII pristine silicon clathrates: A first-principles study. Physical Review B, 2017. 96(24). 